\title{
HIPOTERMIA NO PÓS-OPERATÓRIO IMEDIATO: PERCEPÇÃO DE TÉCNICOS DE ENFERMAGEM
}

\author{
Hypothermia in the immediate post-operative period: perception of nursing technicians
}

\section{Hipotermia en el periodo postoperatorio inmediato: percepción de los técnicos de enfermería} Aline da Silva Sanguiné ${ }^{1}$ D, Gisele Fabiane do Nascimento Ramos² (D), Jaqueline Regina Boschetti ${ }^{3}$ (D) Patricia Treviso ${ }^{4 *}$ (D)

RESUMO: Objetivo: Conhecer a percepção de técnicos de enfermagem que atuam na sala de recuperação pós-anestésica (SRPA) de um hospital filantrópico de grande porte de Porto Alegre sobre hipotermia no pós-operatório imediato (POI). Método: Estudo de campo, exploratório, descritivo e qualitativo. A coleta de dados foi realizada no período de abril a maio de 2017, por meio de entrevista gravada em áudio, utilizando um roteiro composto de 10 questões. Utilizou-se a análise temática para tratamento dos dados. Resultados: Participaram do estudo 19 técnicos de enfermagem. A análise dos dados possibilitou elencar quatro categorias: percepção sobre a definição de hipotermia; complicações da hipotermia para o paciente no POI; condutas de prevenção e reversão de hipotermia; vivências relacionadas a situações de hipotermia. Conclusão: Os entrevistados demonstraram conhecimento sobre os cuidados de enfermagem para prevenção e reversão da hipotermia em pacientes no POI que se encontram na SRPA.

Palavras-chave: Hipotermia. Período pós-operatório. Técnicos de enfermagem. Sala de recuperação.

ABSTRACT : Objective: To understand the perception of nursing technicians working in the post-anesthetic care unit (PACU) of a large philanthropic hospital in Porto Alegre regarding hypothermia in the immediate postoperative period (IPO). Method: an exploratory, descriptive, field study with a qualitative approach. The data collection was performed from April to May 2017, by means of a recorded interview, using a script composed of 10 questions. Thematic analysis was used to treat the data. Results: Nineteen nursing technicians participated in the study. The data analysis resulted in four categories: perception about the definition of hypothermia; complications of hypothermia for the patient in the IPO; actions to prevent and reverse hypothermia; experiences related to situations of hypothermia. Conclusion: The interviewees demonstrated knowledge about nursing care for the prevention and reversal of hypothermia in patients in the IPO in the PACU.

Keywords: Hypothermia. Postoperative period. Licensed practical nurses. Recovery room.

RESUMEN: Objetivo: Comprender la percepción de los técnicos de enfermería que trabajan en la unidad de recuperación postanestésica (URPA) de un gran hospital filantrópico en Porto Alegre sobre la hipotermia en el período postoperatorio inmediato (POI). Método: un estudio exploratorio, descriptivo, de campo con un enfoque cualitativo. La recolección de datos se realizó de abril a mayo de 2017, mediante una entrevista grabada, utilizando un guión compuesto por 10 preguntas. El análisis temático se utilizó para tratar los datos. Resultados: Diecinueve técnicos de enfermería participaron en el estudio. El análisis de los datos dio como resultado cuatro categorías: percepción sobre la definición de hipotermia; Complicaciones de hipotermia para el paciente en el POI; Acciones para prevenir y revertir la hipotermia; Experiencias relacionadas con situaciones de hipotermia. Conclusión: los entrevistados demostraron conocimientos sobre el cuidado de enfermería para la prevención y reversión de la hipotermia en pacientes en la POI en la URPA. Palabras clave: Hipotermia. Periodo posoperatorio. Enfermeros no diplomados. Sala de recuperación.

'Acadêmica do curso de Bacharel em Enfermagem no Centro Universitário Metodista IPA - Porto Alegre (RS), Brasil.

${ }^{2}$ Enfermeira; especialista em Administração dos Serviços de Enfermagem. Enfermeira da Irmandade da Santa Casa de Misericórdia de Porto Alegre (ISCMPA) - Porto Alegre (RS), Brasil.

${ }^{3}$ Enfermeira; especialista em Nefrologia. Enfermeira da ISCMPA - Porto Alegre (RS), Brasil.

`Enfermeira; doutora em Ciências da Saúde; docente do curso de graduação em Enfermagem do Centro Universitário Metodista IPA - Porto Alegre (RS), Brasil.

*Autor correspondente: ptreviso15@gmail.com

Recebido: 21/04/2018 - Aprovado: 01/09/2018

DOI: $10.5327 / 21414-4425201800040006$ 


\section{INTRODUÇÃ̃O}

O período perioperatório é composto de três fases. A primeira é a pré-operatória, que se inicia com a marcação da intervenção cirúrgica e termina com a transferência do paciente para a mesa da sala operatória $(\mathrm{SO})^{1}$; a segunda é a intraoperatória, que inicia com a entrada do paciente na SO e encerrada com a admissão na sala de recuperação pós-anestésica (SRPA); e a terceira é a pós-operatória, que se dá quando o paciente chega à SRPA e termina com a avaliação de acompanhamento no ambiente clínico ou domiciliar ${ }^{1}$.

No período pós-operatório imediato (POI), que compreende as primeiras 24 horas após o procedimento anestésico-cirúrgico, os cuidados de enfermagem visam observar, identificar e conduzir a assistência adequada ao paciente até a recuperação dos efeitos anestésicos, ou seja, até que este esteja apresentando funções motoras e sensoriais normais, orientado, com sinais vitais estáveis, sem evidências de hemorragia e com ausência de complicações ${ }^{2}$.

Nesse período, o paciente pode apresentar diversas complicações decorrentes da anestesia e da cirurgia e também de patologias prévias. Entre as complicações pós-operatórias a que o paciente cirúrgico está sujeito, destaca-se a hipotermia². Grande parte dos pacientes submetidos à anestesia a apresenta, em virtude de alterações na termorregulação provocadas pelo efeito anestésico que pode reduzir até $20 \%$ da produção metabólica de calor ${ }^{3,4}$, mas também por outros fatores, como baixa temperatura da SO, infusão de líquidos frios, perda de sangue, tempo prolongado de cirurgia, entre outros ${ }^{5}$.

A hipotermia é um evento comum no perioperatório ${ }^{6}$. É definida como a redução da temperatura corporal central abaixo de $36^{\circ} \mathrm{C}$. No Brasil, há indicadores de que acomete cerca de $70 \%$ dos pacientes submetidos a processo cirúrgico $^{7}$, podendo ser intencional (terapêutica) - provocada conscientemente pela equipe médica com objetivo de tratamento - ou não intencional (acidental) - que ocorre geralmente em vítimas de trauma, procedimentos cirúrgicos extensos, entre outros ${ }^{8}$.

A hipotermia não intencional em pacientes anestésicocirúrgicos ocorre por causa dos efeitos causados por agentes anestésicos sobre a fisiologia da termorregulação, pela diminuição do metabolismo do paciente e pela exposição ao ambiente frio da SO. Esses fatores podem causar várias complicações ao paciente cirúrgico, por isso, a hipotermia deve ser evitada ou rapidamente tratada ${ }^{9}$. Entre as complicações causadas por ela, destacam-se arritmias cardíacas, anormalidades da coagulação e da função plaquetária, que podem acarretar aumento de perda sanguínea, aumento do risco de mortalidade e de infecção do sítio cirúrgico (ISC), bem como desconforto térmico, aumento na permanência do paciente na SRPA e, consequentemente, aumento do tempo de internaçãó.

O conhecimento e a compreensão sobre a fisiopatogenia, as complicações e as formas de prevenção são importantes e necessárias para que o profissional de enfermagem que atua na SRPA desempenhe seu papel com competência e segurança, contribuindo para a plena recuperação do paciente ${ }^{4,10}$.

O controle da temperatura corporal do paciente no pós-operatório é um desafio para a equipe de enfermagem, que, muitas vezes, tem dificuldades para prestar cuidados em situações de hipotermia. Neste cenário, conhecer a percepção de técnicos de enfermagem poderá contribuir para implementação de melhorias na assistência. Desta forma, buscou-se, neste estudo, aprofundar a temática da hipotermia, visto sua importância na recuperação do paciente no POI. A questão que norteou esta pesquisa foi: Qual é a percepção de técnicos de enfermagem que atuam da sala de recuperação pós-anestésica sobre hipotermia no pós-operatório imediato?

\section{OBJETIVO}

Identificar a percepção de profissionais técnicos de enfermagem que atuam na sala de recuperação pós-anestésica sobre a hipotermia no período pós-operatório imediato.

\section{MÉTODO}

Trata-se de um estudo de campo, exploratório, descritivo, com abordagem qualitativa.

Ele foi realizado em um hospital filantrópico, de grande porte, de Porto Alegre (RS), que presta atendimento a pacientes do Sistema Único de Saúde (SUS), convênios e particulares e conta com uma SRPA com 22 leitos.

Foram convidados a participar do estudo todos os técnicos de enfermagem que trabalhavam, no mínimo, há seis meses na instituição, dos turnos da manhã, tarde, noites I e II na SRPA. Foram excluídos aqueles que estavam em férias, licença saúde ou ausentes da instituição no período de coleta de dados.

Os dados foram colhidos de abril a maio de 2017, após aprovação do projeto de pesquisa pelos Comitês de Ética e Pesquisa (CEP) das instituições proponente (1.908.865) 
e coparticipante (1.940.765). O estudo foi norteado pela Resolução ${ }^{\circ}$ 466 / 2012, do Conselho Nacional de Saúde (CNS).

Os participantes assinaram o Termo de Consentimento Livre e Esclarecido (TCLE) em duas vias, ficando uma com o pesquisador e outra com o participante. Para a realização das entrevistas, utilizou-se um roteiro composto de dez questões, elaborado pelos pesquisadores, abordando os níveis de temperatura considerados normais, a rotina de verificação da temperatura corporal, fatores que podem induzir a hipotermia no período perioperatório, identificação de alterações causadas pelo processo de hipotermia, estratégias para reverter o quadro dessa condição e uma questão para identificar se o participante já vivenciou alguma situação de paciente com hipotermia, como o estado foi identificado e qual foi a conduta empregada. Além das 10 questões, foram realizadas perguntas relativas ao perfil de amostra.

Foi realizado teste piloto com cinco técnicos de enfermagem, objetivando checar a compreensão das questões do instrumento de pesquisa. As entrevistas foram previamente combinadas com cada participante e com a chefia de enfermagem do setor, no intuito de não atrapalhar o andamento das atividades do serviço em questão. Cada entrevista durou, em média, 20 minutos e foi realizada em sala administrativa, respeitando a privacidade dos sujeitos. Elas foram gravadas em áudio e posteriormente transcritas para facilitar a análise.

Para apreciação dos dados, foi utilizada a metodologia de análise temática proposta por Minayo ${ }^{11}$ :

- pré-análise: retomada dos objetivos iniciais da pesquisa, articulando com o material coletado; leitura flutuante, que consiste em uma maior apropriação dos dados, por meio de leitura exaustiva; organização criteriosa do material; e definição de palavras-chave ou categorias;

- exploração do material, que consiste em eleger, classificar e agregar as categorias;

- tratamento dos resultados alcançados, sendo realizada uma correlação dos dados obtidos com a teoria, visando complementar o questionamento do estudo.

\section{RESULTADOS E DISCUSSÃO}

Participaram do estudo 19 técnicos de enfermagem; todos que aceitaram fazer parte dele foram entrevistados. Em relação ao perfil da amostra, a média de idade dos participantes é de 35 anos, a maior parte (17) é do sexo feminino, 10 trabalham também em outra instituição e o tempo médio de formação no curso técnico de enfermagem é de 12 anos. Quanto ao período de atuação na SRPA, um profissional atua há 7 anos e o que tem menos tempo trabalha no setor há 14 meses.

Com base nas respostas dos profissionais que participaram do estudo, foram identificadas as unidades de texto, isto é, frases e palavras que surgem na retórica dos participantes de forma repetida e que inferem uma expressão que as representem $^{12}$. Com base nisso, elencaram-se quatro categorias:

- Categoria 1: percepção sobre a definição de hipotermia;

- Categoria 2: complicações da hipotermia para o paciente no POI;

- Categoria 3: condutas de prevenção e reversão de hipotermia;

- Categoria 4: vivências relacionadas a situações de hipotermia.

Para identificar os participantes da pesquisa, optou-se pela nomenclatura A1 até A19, composta de "A" de anônimo, seguido da numeração correspondente à ordem da realização das entrevistas. As categorias são apresentadas e discutidas na sequência.

\section{Categoria 1: percepção sobre a definição de hipotermia}

No período de recuperação pós-anestésica, o paciente fica aos cuidados da equipe de enfermagem da SRPA, na qual o técnico de enfermagem é um dos profissionais que estará mais próximo dele durante todas as possíveis alterações que podem ocorrer nesse processo, incluindo alterações na termorregulação.

A hipotermia é definida como a perda de calor para o meio, ou seja, o estado em que a temperatura do corpo de um indivíduo está abaixo do valor considerado normal, caracterizada por uma temperatura sanguínea inferior a $36^{\circ} \mathrm{C}$, e está presente nos pacientes submetidos a diferentes cirurgias ${ }^{13}$.

No questionamento sobre a definição de hipotermia, as respostas foram semelhantes em relação ao conceito e enfocam-no como sendo uma situação de "baixa temperatura corporal”, como fica evidente nas seguintes falas: "Hipotermia é a baixa temperatura corporal” (A2); "É a diminuição excessiva da temperatura normal do corpo" (A5).

A hipotermia pode ser responsável por complicações severas no organismo, principalmente no paciente que se encontra no POI. O paciente cirúrgico passa por situações que exigem o bom funcionamento das funções metabólicas, as quais podem ser alteradas pela hipotermia ${ }^{3,4,14}$. 
O organismo humano apresenta uma temperatura corporal central (cérebro, coração, pulmão e órgãos esplâncnicos) entre 36,6 e $37,6^{\circ} \mathrm{C}$. Considera-se hipotermia temperatura menor do que $36^{\circ} \mathrm{C}^{7}$. Quanto aos valores considerados para hipotermia, a maioria dos entrevistados apontou conforme conceito: "Seria, no caso, uma temperatura menor que $36^{\circ}$ " (A9); "Quando estiver menor que 360" (A17).

Dois participantes referem valor diferente dos demais: “TA [temperatura axilar] com valor menor de $34^{\circ}$ " (A1); “34,5" (A2).

O conhecimento dos níveis normais e das alterações da temperatura corporal, por parte da equipe que presta cuidados ao paciente, é fundamental para que medidas de prevenção e reversão sejam aplicadas para garantir qualidade na assistência prestada ${ }^{4,14}$.

Na SRPA, a equipe de enfermagem presta cuidados objetivando recuperação e prevenção de intercorrências decorrentes dos procedimentos anestésico e cirúrgico, por isso são essenciais a verificação e o acompanhamento dos sinais vitais, entre eles a temperatura corporal.

Quando o paciente chega à SRPA, deve-se iniciar o controle dos sinais vitais de 15 em 15 minutos na primeira hora, de 30 em 30 minutos na segunda hora e após de hora em hora, se o paciente permanecer estável ${ }^{9}$. Quanto à rotina de verificação da temperatura na SRPA, todos relataram, conforme amostra a seguir, informações consonantes a isso: "Na primeira hora, de 15 em 15 minutos, na segunda hora, de 30 em 30 minutos e depois de uma em uma hora" (A1); "Na SRPA, verificamos, quando o paciente chega, de 15 em 15 minutos até completar uma hora, depois de 30 em 30, e a partir da terceira hora de uma em uma hora” (A18).

A atuação do profissional da enfermagem no pós-operatório consiste em assistir os pacientes e seus familiares, esclarecer dúvidas e avaliar o seu estado de saúde em todas as fases do tratamento ${ }^{9}$. Ao identificar uma alteração da temperatura corporal, a conduta de enfermagem deve ser rápida, evitando a evolução para um quadro de hipotermia. Os profissionais devem estar atentos a alterações que poderão surgir nessa etapa do POI e estar preparados para intervir de forma segura e com qualidade. Sobre quais fatores podem induzir a hipotermia, foram citados diversos: "Tem vários, um é a duração do tempo de cirurgia, o tipo de cirurgia, de anestesia, estado geral do paciente, né?" (A9); "A cirurgia, num todo, contribui para que o paciente tenha perda de calor do corpo, pouca roupa, medicamentos anestésicos, ambiente da sala frio, perda de sangue, cirurgia demorada” (A19).
Alguns dos entrevistados relataram que a temperatura do ar condicionado, durante o período intraoperatório, interfere na temperatura do paciente, visto que este fica exposto. Além disso, existem outros fatores de risco para a hipotermia, como as cirurgias longas, de médio a grande portes; grande perda sanguínea; alterações causadas pelo procedimento anestésico; antissepsia da pele com agentes químicos frios; paciente totalmente descoberto; infusões de soluções frias; e abertura da cavidade abdominal e/ ou torácica, entre outros $^{3,5,9}$ - todos são justificativas para que toda a equipe fique atenta para a termorregulação do paciente neste período:

"A infusão de líquidos frios IV [intravenosos], a cavidade e feridas abertas, a idade avançada, inalar gases frios, $\mathrm{o}$ ar condicionado da sala cirúrgica com temperatura baixa sendo mais gelada e também os medicamentos anestésicos, são esses que sei” (A16).

A hipotermia não intencional ocorre devido aos efeitos causados pelos agentes anestésicos sobre a fisiologia da termorregulação, pela baixa no metabolismo do paciente e pela exposição ao ambiente frio da $\mathrm{SO}^{3}$. Esses fatores podem causar várias complicações ao paciente cirúrgico, por isso, a hipotermia deve ser evitada ou rapidamente tratada quando manifestada9. Muitos dos entrevistados destacaram: "Acho que seria a alteração no metabolismo pelas drogas usadas na anestesia durante o procedimento, as baixas temperaturas do ambiente e a exposição por muito tempo a essas baixas temperaturas" (A10); "Bom, durante o transoperatório o paciente perde calor e ocorre queda da temperatura, a ação dos medicamentos anestésicos também pode gerar perda de calor; ah, outro fator é a utilização de agentes frios durante a cirurgia” (A11).

A hipotermia pode provocar variadas alterações fisiológicas, como liberação das catecolaminas, pela estimulação simpático-adrenérgica, para o controle da temperatura corporal e aumento da produção de glicose a partir do glicogênio hepático ${ }^{15}$. Na hipotermia, a utilização da glicose é deprimida pela redução da atividade metabólica do fígado, pela inibição da atividade de enzimas e pela redução da produção de insulina no pâncreas; mesmo a administração de insulina é insuficiente para normalizar a glicemia. Algumas falas salientam questões metabólicas: "Acho que os fatores que podem induzir são doenças como diabetes, ambiente frio, problemas de circulação e hipotireoidismo" (A6); "Geralmente a exposição ao frio, níveis baixos de glicose no sangue, medicamentos" (A8).

$O$ controle da temperatura é um aspecto importante para a segurança do paciente. Diante disso, é fundamental 
que as equipes de saúde estejam atentas aos valores de temperatura, bem como aos sinais e sintomas do paciente que chega à SRPA. Salienta-se que a qualificação dos profissionais de enfermagem deve estar fundamentada na problemática do seu processo de trabalho ${ }^{14}$.

\section{Categoria 2: complicações da hipotermia para o paciente no pós-operatório imediato}

O paciente no POI na SRPA fica vulnerável a diversas complicações, que podem atingir diferentes sistemas, principalmente o cardiovascular, o que pode levar a isquemia miocárdica, taquicardia, hipertensão arterial e trombose venosa profunda ${ }^{13}$. Ao perguntar aos participantes se a hipotermia poderia acarretar outras complicações e, se sim, quais seriam elas, as respostas foram diversas: "Sim, pode causar algumas complicações [...] gangrena, arritmia cardíaca, bradicardia, entre outros" (A9); "Acho que sim, pode ocorrer congelamento, morte dos tecidos, danos nos nervos e nos vasos sanguíneos; essa baixa da temperatura corporal também pode causar a diminuição da atividade celular, tendo outras complicações e também causando a morte do paciente" (A12).

A hipotermia pode comprometer também o sistema imunológico, diminuindo a imunidade do paciente, favorecendo a incidência de ISC e o déficit nos fatores de coagulação, como ativação plaquetária e coagulopatia ${ }^{16}$. Um participante relatou: "Sim, e um dos principais fatores de risco é a infecção do sítio cirúrgico" (A6).

Outras complicações são as alterações hidroeletrolíticas, como hipocalemia, hipomagnesemia e hipofosfatemia; e alterações endócrino-metabólicas, tais como baixa no nível de corticoides, queda de insulina, alta resistência periférica de insulina, aumento do TSH (hormônio estimulante da tireoide) e, consequentemente, ampliação de tiroxina e hipoglicemia e hiperglicemia ${ }^{16}$. Afirmação coerente com as seguintes falas: "Congelamento ou morte dos tecidos, gangrena, danos nos nervos e vasos sanguíneos, morte" (A3); "Sim, alteração de consciência, no sensório, extremidades frias" (A7).

Na SRPA, o paciente torna-se vulnerável a diversas complicações, principalmente de origens respiratória, circulatória e gastrintestinal. A hipotermia é classificada em leve (34 a $35,9^{\circ} \mathrm{C}$ ), moderada $\left(30 \text { a } 33,9^{\circ} \mathrm{C} \text { ) e grave (inferior a } 29,9^{\circ} \mathrm{C}\right)^{17}$. Em relação às complicações, foi questionado aos participantes que, se a hipotermia no POI fosse considerada uma alteração fisiológica, como ela seria classificada: em mínima, moderada ou de grande impacto, e qual seria a justificativa. As respostas foram variadas:
- "Na maioria dos pacientes, é mínima, né, pela questão da sala, pela questão da perda sanguínea, mas tem a moderada também, que é de acordo com os anestésicos, a anestesia influencia bastante na temperatura. [...]” (A2);

- "Se fosse alteração fisiológica, seria de moderada a mínima, porque geralmente os pacientes chegam na sala de recuperação pós-anestésica com temperatura de $35,5^{\circ \prime}$ (A6).

Na hipotermia moderada, o paciente pode apresentar sintomas de arritmias atriais, redução da frequência cardíaca, alteração no nível de consciência, redução da frequência respiratória, depressão do reflexo de deglutição, dilatação das pupilas, redução dos tremores, hiporreflexia, hipotensão e possíveis alterações no eletrocardiograma ${ }^{16}$. Observam-se as seguintes respostas: "A meu ver, seria moderada, podendo causar sangramentos na FO [ferida operatória], disfunção respiratória" (A7); "Acredito que seja moderada, devido às complicações causadas no pós-operatório" (A9).

Quando a temperatura se encontra menor do que $30^{\circ} \mathrm{C}$, é considerada de grande impacto, pois aumenta os riscos de o paciente entrar em estado de coma, apneia, pupilas não reativas, oligúria, arritmias ventriculares, assistolia, podendo inclusive ir a óbito ${ }^{16}$. Cientes de tais conhecimentos, alguns entrevistados salientam: "Pra mim, classifico de grande impacto, pois, tendo a diminuição da atividade celular, vai interferir na cicatrização da FO e da recuperação anestésica" (A11); "Grande impacto. Porque um dos primeiros cuidados no pós-operatório é manter o paciente aquecido, para evitar a diminuição da atividade do paciente" (A13).

Quanto aos fatores que podem induzir à hipotermia, ressaltam-se os agentes anestésicos, a temperatura do ambiente, o tempo de exposição do paciente em locais com baixa temperatura, a administração de infusões venosas frias, os distúrbios sistêmicos, entre outros. Nesse parâmetro, foram citados alguns fatores, conforme o conhecimento de cada entrevistado: "Pode repetir, peraí $[s i c][\ldots]$ vasoconstrição, atraso na cicatrização, desconforto geral do paciente né” (A1).

Quanto aos sinais de alterações de temperatura, foram caracterizados: "Pele fria e pálida, muitos calafrios, temperatura marcando abaixo de $36^{\circ} \mathrm{C}$, extremidades azuladas" (A4); "Bom, o paciente fica com tremores incontroláveis, pulsação fraca, tem dificuldades de controlar os movimentos do corpo" (A18).

Sendo assim, a monitorização da temperatura durante o ato anestésico no transoperatório é de grande importância, 
pois proporciona a detecção precoce da hipotermia e/ ou de complicações, podendo facilitar o controle térmico no POI do paciente na SRPA ${ }^{18}$.

\section{Categoria 3: condutas de prevenção e reversão de hipotermia}

Saber identificar, conhecer e compreender os cuidados em uma situação de hipotermia é de grande importância para que o profissional tenha condições de assistir o paciente, prestando cuidados com qualidade, segurança e eficácia. O aquecimento no perioperatório reduz a perda sanguínea, proporcionando redução de dor, tremores e risco de ISC no POI ${ }^{19}$.

Quanto às medidas de prevenção de hipotermia, os participantes apontam que fazer uso de artifícios de aquecimento do paciente é a principal conduta de enfermagem para a prevenção e reversão da hipotermia:

- "Fazer o aquecimento do paciente desde a sala cirúrgica com cobertores e manta térmica, pode se usar também algodão laminado nos membros inferiores, infundir soluções aquecidas, aquecer a cabeça com toucas, monitorar temperatura" (A11);

- "Então, [...] quando o paciente chega pra nós, já colocamos cobertores neles, e manter a temperatura ambiente na SRPA" (A14).

Os cuidados para manutenção da normotermia do paciente se dão por meio da monitorização da temperatura corporal como principal intervenção de enfermagem para prevenir e diminuir riscos de complicações futuras ${ }^{6}$. Diante das medidas para prevenção da hipotermia, os participantes apontam que aquecer o paciente é cuidado estratégico: "A principal é aquecer o paciente" (A5); "Aquecer de imediato e manter o termômetro no monitor para controle rígido da temperatura" (A6).

Outras estratégias ainda podem ser utilizadas, tanto de aquecimento ativo como passivo. O aquecimento passivo é um método de baixo custo e eficaz, que consiste em cobrir e aquecer o paciente com lençóis, cobertores ou mantas de algodão, podendo ainda ser usado um envoltório nos membros inferiores com algodão laminado e atadura de crepe. Nas medidas ativas de aquecimento, utilizam-se cobertor elétrico, manta térmica, colchão de água aquecida, irrigação e infusão venosa com fluidos aquecidos, entre outros. Todos esses métodos para o aquecimento do paciente podem reduzir a perda de calor em $30 \%$ durante a cirurgia e na SRPA ${ }^{14,18}$.

\section{Categoria 4: vivências relacionadas às situações de hipotermia}

O roteiro de entrevista foi finalizado com uma questão sobre a experiência pessoal de cada participante quanto a uma situação de hipotermia, como foi identificada e qual conduta de enfermagem foi realizada na ocasião. Todos os participantes relataram já ter vivenciado alguma situação de cuidado com paciente hipotérmico: "Sim, paciente chegou com tremores, calafrios, extremidades geladas e roxeadas. Cobri ele com dois cobertores, coloquei manta térmica em 43 graus para aquecer rápido e luvas com água quente sobre os pés" (A14); "Sim, paciente chegou tremendo muito, extremidades frias, frequência cardíaca lenta e respiração também, pele pálida. Foi aquecido com cobertores e com a manta térmica em 43 graus." (A19)

Alguns entrevistados relataram não ter vivido nenhuma situação de hipotermia grave, mas sinalizaram de que forma conduziriam a questão: "Uma situação bem grave não vivi, mas, como falei, se tivesse ocorrido, avisaria a enfermeira da SRPA e providenciaria cobertores, uma manta térmica para reverter a situação rápido" (A12); "Uma situação grave nunca presenciei, mas pelos sintomas de tremor, de extremidades geladas, TA baixa, iria providenciar cobertores para seu aquecimento" (A18).

O fator de todos os participantes relatarem ter vivenciado alguma situação de cuidado a paciente com quadro de hipotermia denota o quanto essa complicação é comum no POI e a importância de a equipe de enfermagem utilizar estratégias de cuidado durante o período perioperatório, visando à prevenção dessa circunstância adversa, assim como a necessidade de reversão precoce quando ela ocorre.

\section{CONCLUSÃO}

O estudo possibilitou identificar a percepção de profissionais técnicos de enfermagem que atuam na SRPA sobre a hipotermia no POI, demonstrando que os participantes têm conhecimento quanto a definição dessa condição, valores que a determinam e principais fatores que contribuem para desencadeá-la. Nas situações em que a hipotermia pode acarretar complicações ao paciente no POI, os entrevistados apontaram sintomas como diminuição da atividade celular, alterações fisiológicas e metabólicas, ISC, entre outros, que podem evoluir para uma situação sem reversão da hipotermia.

Os achados apontam que os participantes utilizam diversas estratégias de cuidado para prevenir e reverter a hipotermia 
no POI, tanto com a utilização de aquecimento passivo como de aquecimento ativo.

Neste contexto, acredita-se que o esclarecimento sobre a percepção dos técnicos de enfermagem em relação à hipotermia possa fornecer subsídios para estimular a busca e o aperfeiçoamento de novos conhecimentos, assim como evidenciar a importância da assistência de enfermagem no período perioperatório no que concerne à prevenção e reversão de complicações no período pós-operatório, promovendo novos estudos nessa área.

\section{REFERÊNCIAS}

1. Smeltzer SC, Bare BG, Hinkle JL, Kleever KH. Brunner \& Suddarth: tratado de enfermagem médico-cirúrgica. $12^{\mathrm{a}}$ ed. Rio de Janeiro: Guanabara Koogan; 2011.

2. Associação Brasileira de Enfermeiros de Centro Cirúrgico, Recuperação Anestésica e Centro de Material e Esterilização. Diretrizes de práticas em enfermagem cirúrgica e processamento de produtos para a

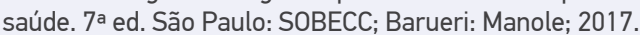

3. Lopes IG, Magalhães MAS, Sousa ALA, Araújo IMB. Prevenir a hipotermia no perioperatório: revisão integrativa da literatura. Rev Enfer Ref. 2015;4(4):147-55. http://dx.doi.org/10.12707/RIV14027

4. Dutra LCB, Meneses RO. Avaliação da hipotermia não intencional em sala de recuperação anestésica. Rev Rede Cuid Saúde. 2017;11(1):1-15.

5. Ribeiro MB, Peniche, AC, SilvaSCF. Complicações na sala de recuperação anestésica, fatores de risco e intervenções de enfermagem: revisão integrativa. Rev SOBECC. 2017;22(4):218-29. http://dx.doi.org/10.5327/ Z1414-4425201700040007

6. Poveda VB, Martinez EZ, Galvão CM. Métodos ativos de aquecimento cutâneo para a prevenção de hipotermia no período intraoperatório: revisão sistemática. Rev Latino-Am Enferm. 2012;20(1):1-9. https:// doi.org/10.1590/S0104-11692012000100024

7. Muniz GS, Teles NSB, Leitão IMTA, Almeida PC, Leitão MC. Hipotermia acidental: implicações para os cuidados de enfermagemno transoperatório. RevSOBECC. 2014;19(2):79-86. http://dx.doi.org/10.4322/sobecc2014.009

8. Ribeiro E, Navarro NT, Armede VCB, Rodrigues HS, Valle JP, Duran ECM. Frequência de hipotermianão intencional de cirurgias eletivas. RevSOBECC. 2016;21(2):68-74. https://doi.org/10.5327/Z1414-442520160002000

9. Carvalho R, Bianchi ERF. Enfermagem em centro cirúrgico e recuperação. $2^{\text {a }}$ ed. São Paulo: Manole; 2016.

10. Mendoza IYQ, Peniche ACG, Puschel VAA. Conhecimento sobre hipotermia dos profissionais de enfermagem do centro cirúrgico.
Rev Esc Enferm USP. 2012;46(4):123-9. http://dx.doi.org/10.1590/ S0080-62342012000700018

11. Minayo MCS. 0 desafio do conhecimento: pesquisa qualitativa em saúde. 12a ed. São Paulo: Hucitec; 2010.

12. Caregnato RCA, Mutti R. Pesquisa qualitativa: análise de discurso versus análise de conteúdo. Texto Contexto Enferm. 2006;15(4):67984. http://dx.doi.org/10.1590/S0104-07072006000400017

13. Mattia AL, Barbosa MH, Rocha AM, Farias HL, Santos CA, Santos DM. Hipotermia em pacientes no período perioperatório. Rev Esc Enferm USP. 2012;46(1):60-6. http://dx.doi.org/10.1590/ S0080-62342012000100008

14. Souza A, Palazzo S, Montezello D. Conhecimentos dos profissionais de enfermagem de centro cirúrgico sobre hipotermia em pacientes cirúrgicos oncológicos. Rev SOBECC. 2017;22(4):188-92. https://doi. org/10.5327/Z1414-4425201700040003

15. Pestel GJ, Kurz A. Hypothermia - it's more than a toy. Anestesiol. 2005;18:151-6.

16. Biazzotto CB, Brudniewski M, Schmidt AP, Auler Júnior JOC. Perioperative hypothermia. Rev Bras Anestesiol. 2006;56(1):89-106.

17. Pereira NHC, Rocha ADM, Mattia ALD. Infusão venosa aquecida relacionada à prevenção das complicações da hipotermia intraoperatória. Rev SOBECC. 2014;19(2):74-8. http://dx.doi. org/10.4322/sobecc2014.013

18. Danczuk RFT, Nascimento ERP, Silveira NRH, Hermida PMV, Rasia AR. Métodos de aquecimento na prevenção da hipotermia no intraoperatório de cirurgia abdominal eletiva. Esc Anna Nery. 2015;19(4):578-84. http://dx.doi.org/10.5935/1414-8145.20150077

19. Nascimento PDF, Bredes AC, Mattia ALD. Complicações em idosos em sala de recuperação pós-anestésica. Rev SOBECC. 2015;20(2):6472. https://doi.org/10.5327/Z1414-4425201500020002 